\title{
Spirometric Values of Patients with Chronic Obstructive Pulmonary Disease in Dr. Hasan Sadikin General Hospital Bandung
}

\author{
Christopher Rinaldi, ${ }^{1}$ Rudolf Andean, ${ }^{2}$ Irma Ruslina Defi ${ }^{3}$ \\ ${ }^{1}$ Faculty of Medicine Universitas Padjadjaran, Indonesia, ${ }^{2}$ Department of Biomedical Sciences \\ Faculty of Medicine Universitas Padjadjaran, Indonesia, ${ }^{2}$ Department of Physical Medicine and \\ Rehabilitation Faculty of Medicine Universitas Padjadjaran/Dr. Hasan Sadikin General Hospital \\ Bandung, Indonesia
}

\section{Abstract}

Background: Chronic obstructive pulmonary disease (COPD) is a frequently underdiagnosed disease. Definitive COPD has been diagnosed using spirometry to evaluate pulmonary function. This study aimed to explore the spirometric values of COPD patients to aid in the diagnosis of COPD.

Methods: A descriptive study was conducted in Dr. Hasan Sadikin General Hospital Bandung from October to November 2015. Data on patients diagnosed with COPD, aged 40 to 60 years old, were collected during the year 2014 prior to ethical clearance. Spirometry values consisted of forced expiratory volume in one second (FEV1), forced vital capacity (FVC), and FEV1/FVC ratio were taken from the medical records.

Results: Sixty-eight COPD patients, consisting of 48 males and 20 females, were included of with most were classified as moderate COPD. The FEV1 was $49.72 \pm 17.79$, whereas the FVC was 55.90 (range 22.80-96.03), and the FEV1/FVC ratio was $0.70 \pm 0.12$. Most patients were classified into moderate COPD who had FEV1 between 50 and $80 \%$ of normal as GOLD 2 standard.

Conclusions: Even though patients seem to have no symptoms, spirometric values may show a silent insufficient lung function affecting patients' daily life.

Keywords: Chronic obstructive pulmonary disease, COPD, FEV1, FVC, spirometry

\section{Introduction}

Global Initiative for Chronic Obstructive Lung Disease (GOLD) has defined chronic obstructive pulmonary disease (COPD) as a chronic, persistent, and progressive airway limitation, that is associated with enhanced inflammatory response and possible excacerbation with exposure to noxious particles or gases. ${ }^{1,2}$ Epidemiological studies have shown that COPD has a high burden and the prevalence is relatively increased. Prevalence is higher in studies involving older patients over 40 years old $(9.0 \%)$, smokers $(15.4 \%)$, males (9.8\%), and individuals living in urban areas $(10.2 \%)$. Interestingly, COPD is frequently a symptomatic in the early stages. It is shown in the study that reported a much lower estimated prevalence of patient-reported
COPD (4.9\%) compared to COPD diagnosed by spirometric criteria $(9.2 \%){ }^{3}$ The disease may cause physical disabilities by reduced activities of daily living (ADL), instrumental activities of daily living (IADL), and mobility disability (MD). Disability aside, COPD remains incurable and the best treatments may only be able to inhibit the disease's progression. ${ }^{4}$

A survey by the Epidemiology and Impact of COPD (EPIC) Asia, conducted in 2012 in North Asia countries (China, Hong Kong, and Taiwan) and Southeast Asia countries (Indonesia, Malaysia, the Philippines, Singapore, Thailand, and Vietnam) has shown that several Southeast Asian countries have a worse health condition for patients with COPD and a greater rate of underdiagnosis of COPD compared to North Asian countries. ${ }^{5}$ The survey result is concurrent with the existing challenges in

Correspondence: Christopher Rinaldi, Faculty of Medicine Universitas Padjadjaran, Jalan Raya Bandung Sumedang Km. 21 Jatinangor, Sumedang Indonesia , E-mail: chrinaldi93@yahoo.com 
diagnosing COPD. Despite being a possible cause for disabilities and the tendency for rapid progression of the disease's severity, COPD is a frequently underdiagnosed and misdiagnosed condition. Therefore, solutions have been aimed at making earlier diagnosis and management to improve the patients' condition and to delay with the progression of the disease. These efforts may be able to reduce hospitalization and exacerbation rates. ${ }^{6}$

Spirometry is an essential tool, and also serves as a gold standard in diagnosing COPD. It contains several pulmonary function values such as forced expiratory volume in one second (FEV1) and forced vital capacity (FVC) as main spirometric values that require an assessment to determine the diagnosis and the severity of COPD to give an appropriate management plan. ${ }^{7}$ This study aimed to obtain a description of the spirometric values of patients with COPD that may aid the diagnosis of COPD.

\section{Methods}

This study was conducted from October to November 2015. All data on COPD patients during 2014 were collected from medical records, including patients who underwent pulmonary function tests with spirometry in the Department of Respirology, Dr. Hasan Sadikin General Hospital Bandung. Inclusion criteria were patients diagnosed with COPD and the age range of 40 years to 60 years old. Exclusion criteria were patients diagnosed with acute exacerbation of COPD or diagnosed with other illnesses secondary to COPD.

The data consisted of basic characteristics consisted of age, sex, height, weight, body mass index (BMI) and disease severity according to the Global Initiative for Chronic Obstructive Lung Disease (GOLD) system. The GOLD guideline was used as a combined COPD assessment approach to group patients according to symptoms and previous history of exacerbations and the severity of COPD was categorized according to GOLD criteria. A GOLD criterion was based on spirometry results such as FEV1, FVC, and FEV1/FVC ratio. The criteria were divided into 4 groups according to percent predicted FEV1 with GOLD1 being the least severe and GOLD 4 being the most severe. In patients with FEV1/FVC $<0.70$. GOLD criteria was applied, that were GOLD 1-mild: FEV1 $\geq 80 \%$ predicted, GOLD 2moderate: $50 \% \leq$ FEV $1<80 \%$ predicted, GOLD 3-severe: $30 \% \leq \mathrm{FEV} 1<50 \%$ predicted, GOLD 4 -very severe: FEV1 $<30 \%$ predicted. The study protocol was granted from the Health
Research Ethics Committee, Dr. Hasan Sadikin General Hospital Bandung No. 113/TB/RSHS/ Nov15.

\section{Results}

In total, sixty-eight data on spirometric values and physical profiles of COPD patients were collected, including 48 male and 20 female COPD patients. The basic characteristic of COPD patients was shown in Table 1. The majority of patients $(44.1 \%)$ had been classified as GOLD 2 or moderate COPD (Table 1 ).

Across all spirometric values, male patients tended to have a higher score compared to female patients. Despite the higher predicted and tested spirometric values, the percent predicted spirometric values of male COPD patients were lower compared to the percent predicted spirometric values of female COPD patients. However, no statistical measurement had been conducted.

The mean FEV1/FVC ratios were mostly around 0.7 that indicated minimal obstructive respiratory problems despite being diagnosed as COPD that required the ratio to be below 0.7. Several samples from male and female COPD patients were found to have a higher FEV1/FVC ratio than 0.7 , again no statistical analyses had been performed (Table 2).

\section{Discussion}

The COPD is increasing, predominantly in male as shown in our study. However, in most developed countries, there is a shifting of COPD prevalence towards females. ${ }^{8}$ This occurrence may be attributed to several factors. Women are less tolerant to the effects of smoking, and these effects are limited only on lower usage of cigarettes. As for more than 20 cigarettes a day, the dose-response relationship is similar to men. ${ }^{9}$ Another alternative explanation for this proportion is the estimated prevalence of smoking women in Indonesia. According to survey data obtained from 1980 to 2012, Indonesia has estimated prevalence rates of daily smoking greater than $40 \%$ for males and less than $5 \%$ for females. ${ }^{10}$ The difference in prevalence between sexes may justify the view that COPD is a predominantly male disease. ${ }^{9}$ Interestingly, survey data and modeling by WHO conducted in the subregion of Southeast Asia region B, which is consisted of Indonesia, Thailand, and Sri Lanka, has reported an estimated proportion of $56 \%$ females that are exposed to second hand smoke, compared to $35 \%$ females exposed to second hand smoke 
Table 1 Basic Characteristic of Chronic Obstructive Pulmonary Disease Patients at Dr. Hasan Sadikin General Hospital 2014

\begin{tabular}{|c|c|c|c|}
\hline Characteristics & Male $(n=48)$ & Female $(n=20)$ & Total $(n=68)$ \\
\hline \multicolumn{4}{|l|}{ Age (years) } \\
\hline $40-45$ & 9 & 4 & 13 \\
\hline $46-50$ & 5 & 4 & 9 \\
\hline $51-55$ & 18 & 6 & 24 \\
\hline $56-60$ & 16 & 6 & 22 \\
\hline \multicolumn{4}{|l|}{ Height (meter) } \\
\hline$<1.50$ & 3 & 9 & 12 \\
\hline $1.50-1.60$ & 20 & 11 & 31 \\
\hline$>1.60$ & 25 & - & 25 \\
\hline \multicolumn{4}{|l|}{ Weight (kilograms) } \\
\hline$<50$ & 13 & 10 & 23 \\
\hline $50-59$ & 16 & 6 & 22 \\
\hline $60-70$ & 13 & 4 & 17 \\
\hline$>70$ & 6 & - & 6 \\
\hline \multicolumn{4}{|l|}{ BMI (kilograms/meter $\left.{ }^{2}\right)^{*}$} \\
\hline$\leq 18.5$ & 11 & 6 & 17 \\
\hline $18.5-24.9$ & 26 & 9 & $35^{*}$ \\
\hline $25.0-29.9$ & 7 & 4 & 11 \\
\hline$\geq 30.0$ & 4 & 1 & 5 \\
\hline \multicolumn{4}{|l|}{ Severity } \\
\hline GOLD 1 & 3 & 3 & 6 \\
\hline GOLD 2 & 23 & 7 & $30^{*}$ \\
\hline GOLD 3 & 13 & 6 & 19 \\
\hline GOLD 4 & 9 & 4 & 13 \\
\hline
\end{tabular}

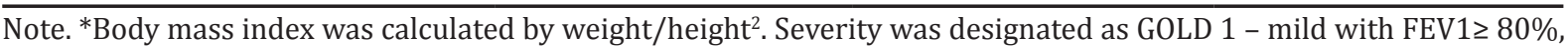
GOLD 2 - moderate with 50\% $\leq \mathrm{FEV} 1<80 \%$, GOLD 3 - severe with $30 \% \leq \mathrm{FEV} 1<50 \%$ and GOLD 4 - very severe with FEV1 $<30 \%$.

worldwide. $^{11}$ The combination of earlier described factors may contribute to the development of COPD in women, although it is not higher than men, which may indicate the beginning of a shifting paradigm of patients with COPD in Indonesia.

The age range of 40 years to 60 years old included in this study is to minimize the effects of aging and possible undiagnosed comorbidities. The aging process contributes to the reduction of FEV1 and FVC in the elderly population and it will increase false positivities that tends to occur from the normal aging process, leading to overdiagnosis of COPD in the middle-aged and elderly population. The spirometric values observed in this study have shown a decline in older age groups. This may be caused by the natural decline of lung function and increased bronchial responsiveness. Lung function peaks between 20 to 35 years old and follows a steady decline after 35 years. The rate of decline varies individually and it has shown a nonlinear pattern with the estimated rate of decline around $25 \mathrm{ml} /$ year; starting at age 35 to 40 years old and may increase to $60 \mathrm{ml} /$ year after the age of 70 years. Such a natural decline of lung function among very old people may be mistaken for COPD during spirometry. Furthermore, bronchial responsiveness is measured by the dose of 
Table 2 Spirometric Values of Chronic Obstructive Pulmonary Disease Patients at Dr. Hasan Sadikin General Hospital 2014

\begin{tabular}{|c|c|c|c|}
\hline & Male & Female & Total \\
\hline \multicolumn{4}{|l|}{ FEV1* } \\
\hline 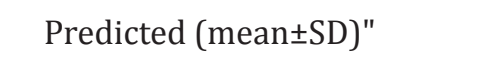 & $2.68+0.34$ & $2.05+0.19$ & $2.49+0.42$ \\
\hline Result (mean \pm SD)" & $1.33+0.53$ & $1.03+0.47$ & $1.24+0.53$ \\
\hline $\mathrm{PP}^{* *}($ mean $\pm \mathrm{SD})$ & $49.50+18.09$ & $50.26+20.86$ & $49.72+18.79$ \\
\hline \multicolumn{4}{|l|}{$\mathrm{FVC}^{* * *}$} \\
\hline Predicted (Median; min-max) & $3.45 ; 2.55-4.07$ & $2.48 ; 1.92-2.67$ & $3.37 ; 1.92-4.07$ \\
\hline Result (Median; min-max) & $1.97 ; 0.76-2.78$ & $1.22 ; 0.70-2.42$ & $1.76 ; 0.70-2.78$ \\
\hline PP (Median; min-max) & $56.20 ; 22.80-79.20$ & $52.42 ; 27.89-96.03$ & $55.90 ; 22.80-96.03$ \\
\hline FEV1/FVC $* * * *(m e a n \pm S D ")$ & $0.68+0.13$ & $0.73+0.12$ & $0.70+0.12$ \\
\hline
\end{tabular}

Note: *FEV1=Forced expiratory volume in one second in liters, ** $\mathrm{PP}=$ percent predicted of each values in percentages, *** FVC=Forced vital capacity in liters, ${ }^{* * * * F E V 1 / F V C=f o r c e d ~ e x p i r a t o r y ~ v o l u m e ~ i n ~ o n e ~ s e c o n d / f o r c e d ~ v i t a l ~ c a p a c i t y ~}$

methacholine required to cause a significant reduction in FEV1, which is about $20 \%$ from the baseline. Since methacholine can cause bronchospasm, lower doses indicate higher bronchial responsiveness. Younger people and older people are much more susceptible to FEV1 reduction from methacholine. ${ }^{12-14}$ Bronchial hyper responsiveness may be a confounding factor in COPD, especially if the reduction in the flow rate, FEV1 specifically, is reversible. Therefore, the older age groups on the population might be excluded in this study to minimize the effect of the natural decline in lung function due to aging and bronchial hyperresponsiveness.

Moreover, COPD is uncommon under the age of 40 years old. The predisposition of COPD in young adults may be attributable to other causes such as chronic lung disease of prematurity (CLDP) and bronchopulmonary dysplasia (BPD). There are individuals who may have a lower FEV1/FVC ratio as their normal lung function. Young individuals who have developed COPD will most likely have CLDP as a secondary diagnosis instead of the main issue. Issues that occur during growth and development may be recognized as the main issues instead the respiratory disease itself.

The hazards include early life viral infection, allergic sensitization, and prematurity. Hazards during the developmental period of infants may produce lower lung function in later life, thus, predisposing them to develop an obstructive disease phenotype that may be diagnosed as COPD as the main diagnosis. ${ }^{15}$ Amore extensive history taking is required especially history during the younger ages to exclude other differential diagnoses. Therefore, the exclusion of younger ages on the population has been made.

By definition, COPD involves chronic and persistent airway obstruction. The symptoms of COPD range from asymptomatic to the ones that may significantly reduce the quality of life. The airway obstruction in COPD is mainly diagnosed using spirometers and the most commonly used diagnostic criteria for COPD were the GOLD criteria. Measuring the severity of COPD using post-bronchodilator with FEV1/FVC ratio below 0.7 , then continued by the assessment of percent predicted FEV1 indicates a partially irreversible airway obstruction. ${ }^{2}$ The cut off point is not absolute. Interestingly, several patients in this study have FEV1/FVC above 0.7 and yet, still diagnosed as COPD. Several alternatives can be used in assessing the lower limit of normal (LLN) by using body plethysmography and diffusion capacity tests.

A study has compared LLN and fixed FEV1/ FVC ratio in terms of positive predictive value and negative predictive value, resulting in no significant differences between the two cutoff points. ${ }^{16}$ Reference value for LLN is adjusted according to age and defined as the lower fifth percentile of the population in FEV1/ FVC ratios as abnormal. ${ }^{17}$ The LLN claims to be able to better diagnose the condition by reducing misdiagnosis of COPD, however, the LLN has proven to be comparably minimal to the fixed FEV1/FVC ratio that GOLD has proposed. ${ }^{16}$ Moreover, the LLN is relatively more difficult to apply in practical situations 
and primary healthcare settings compared to the fixed ratio. ${ }^{16}$ The findings of COPD outside of the currently established reference value may represent the difficulty in establishing the diagnosis of COPD, leading to mislabelling of the disease. Spirometry can only be described as a tool for diagnosis. The true gold standard for COPD diagnosis lies in the diagnostic criteria used to interpret the spirometry results to an accurate clinical diagnosis.

So far, spirometry has been used as a gold standard for the diagnosis of COPD and many other restrictive or obstructive respiratory disorders. A diagnostic tool, even as a gold standard, will only display desired effectiveness if the operators were competent in its usage. Misdiagnosis of COPD is not an uncommon occurrence. According to a prospective, controlled, trial study conducted in Italy, about $30.1 \%$ of patients diagnosed with COPD have a normal spirometry result. The definitive diagnosis of COPD using spirometry has been found with large variability, ranging from 0 to $100 \%$, with a median of $37 \%{ }^{18}$ Misclassification of COPD may result in mismanagement of patients, resulting from further in a faster lung function decline in patients.

The limitations of the study include the lack of other variables in medical records, such as smoking history and post-bronchodilator spirometric values. Smoking history is one of the most important risk factors in COPD development. Early identification of COPD is important because smoking cessation at an early stage of COPD is the only intervention that substantially improves the prognosis by normalizing lung function decline and decreasing morbidity and all-cause mortality.

Confounding factors of the study include the assessment of spirometry adequacy. The study has not been able to directly evaluate the operators or the physicians in performing the spirometry and also patients' effort during the spirometry which may influence the diagnosis and the spirometric values recorded by the spirometer.

Mislabelling of patients without COPD in clinical practice is an issue that needs to be addressed. Therefore, there are several suggestions in this study aiming to provide possible solutions. Training in spirometry usage for physicians and attendants may be beneficial in improving diagnostic accuracy in COPD. Since spirometry is a tool, it requires calibration to perform optimally.

This study has shown that COPD in male patients tend to have more severe form compared to women. Furthermore, there are still patients diagnosed with COPD despite the normal FEV1/FVC ratios. COPD is an incurable disease and the best way to manage such disease is to prevent the disease from occurring in the first place.

To conclude, even though patients seem to have no symptoms, spirometric values may show a silent insufficient lung function affecting patients' daily life.

\section{References}

1. Viegi G, Pistelli F, Sherrill DL, Maio S, Baldacci S, Carrozzi L. Definition, epidemiology and natural history of COPD. Eur Respir J. 2007;30(5):993-1013.

2. Global Initiative for Chronic Obstructive Lung Disease. Global strategy for the diagnosis, management and prevention of COPD (updated 2015). Fontana: GOLD [internet] 2015 [Cited 2015 October 12]. Available from: http://www.goldcopd.org.

3. Halbert RJ, Natoli JL, Gano A, Badamgarav E, Buist AS, Mannino DM. Global burden of COPD: systematic review and metaanalysis. Eur Respir J. 2006;28(3):523-32.

4. Rodriguez-Rodriguez P, Jimenez-Garcia R, Hernandez-Barrera V, Carrasco-Garrido P, Puente-Maestu L, Miguel-Diez JD. Prevalence of physical disability in patients with chronic obstructive pulmonary disease and associated risk factors. COPD. 2013;10(5):611-7.

5. Lim S, Lam DC-L, Muttalif AR, Yunus F, Wongtim S, Lan LTT, et al. Impact of chronic obstructive pulmonary disease (COPD) in the Asia-Pacific region: the EPIC Asia population-based survey. Asia Pac Fam Med. 2015;14(1):4.

6. Fromer L. Diagnosing and treating COPD: understanding the challenges and finding solutions. Intl J Gen Med. 2011;4:729-39.

7. BaileyKL. TheImportance oftheAssessment of Pulmonary Function in COPD. Med Clin North Am. 2012;96(4):745-52.

8. Aryal S, Diaz-Guzman E, Mannino DM. COPD and gender differences: an update. Transl Res. 2013;162(4):208-18.

9. Sørheim I, Johannessen A, Gulsvik A, Bakke P, Silverman E, DeMeo D. Gender differences in COPD: are women more susceptible to smoking effects than men?. Thorax. 2010;65(6):480-85.

10. Ng M, Freeman MK, Fleming TD, Robinson M, Dwyer-Lindgren L, Thomson B, et al. Smoking prevalence and cigarette consumption in 187 countries, 1980- 
2012. JAMA. 2014;311(2):183-92.

11. Öberg M, Jaakkola MS, Woodward A, Peruga A, Prüss-Ustün A. Worldwide burden of disease from exposure to second-hand smoke: a retrospective analysis of data from 192 countries. Lancet. 2011;377(9760):139-46.

12. Schermer TRJ, Smeele IJM, Thoonen BPA, Lucas AEM, Grootens JG, van Boxem TJ, et al. Current clinical guideline definitions of airflow obstruction and COPD overdiagnosis in primary care. Eur Respir J. 2008;32(4):945-52.

13. Sharma G, Goodwin J. Effect of aging on respiratory system physiology and immunology. Clin Interv Aging. 2006;1(3): 253-60.

14. Jarad N. Chronic obstructive pulmonary disease (COPD) and old age?. Chron Respir Dis. 2011;8(2):143-51.

15. Martinez FD. The origins of asthma and chronic obstructive pulmonary disease in early life. Proc Am Thorac Soc. 2009;6(3):272-7.

16. Hoesein FAM, Zanen P, Sachs AP, Verheij TJ, Lammers JW, Broekhuizen BD. Spirometric thresholds for diagnosing COPD: 0.70 or LLN, pre- or post-dilator values?. COPD. 2012;9(4):338-43.

17. Hansen JE, Sun XG, Wasserman K. Spirometric criteria for airway obstruction: use percentage of FEV1/FVC ratio below the fifth percentile, not $<70 \%$. Chest. 2007;131(2):349-55.

18. Lusuardi M, De Benedetto F, Paggiaro P, Sanguinetti CM, Brazzola G, Ferri P, et al. A randomized controlled trial on office spirometry in asthma and COPD in standard general practice: data from spirometry in Asthma and COPD: a comparative evaluation Italian study. Chest. 2006;129(4):844-52. 